\title{
Phase noise reduction of a semiconductor laser in a composite optical phase-locked loop
}

\author{
Naresh Satyan \\ Jacob Sendowski \\ California Institute of Technology \\ Department of Electrical Engineering \\ 1200 East California Boulevard 136-93 \\ Pasadena, California 91125 \\ Email: naresh@caltech.edu
}

\author{
Arseny Vasilyev \\ California Institute of Technology \\ Department of Applied Physics \\ 1200 East California Boulevard 128-95 \\ Pasadena, California 91125
}

\section{George Rakuljic}

Telaris Incorporated

2118 Wilshire Boulevard \#238

Santa Monica, California 90403

\author{
Amnon Yariv \\ California Institute of Technology \\ Department of Applied Physics \\ and \\ California Institute of Technology \\ Department of Electrical Engineering \\ 1200 East California Boulevard 128-95 \\ Pasadena, California 91125
}

\begin{abstract}
The bandwidth and residual phase noise of optical phaselocked loops (OPLLs) using semiconductor lasers are typically constrained by the nonuniform frequency modulation response of the laser, limiting their usefulness in a number of applications. It is shown in this work that additional feedback control using an optical phase modulator improves the coherence between the master and slave lasers in the OPLL by achieving bandwidths determined only by the propagation delay in the loop. A phase noise reduction by more than a factor of two is demonstrated in a proof-of-concept experiment using a commercial distributed feedback semiconductor laser. (C) 2010 Society of Photo-Optical Instrumentation Engineers. [DOI: 10.1117/1.3518077]
\end{abstract}

Subject terms: optical phase-locked loops; semiconductor lasers; frequency modulation response; optical phase modulators; coherence.

Paper 100682R received Aug. 23, 2010; revised manuscript received Sep. 30, 2010; accepted for publication Oct. 12, 2010; published online Dec. 6, 2010.

\section{Introduction}

Optical phase-locked loops (OPLLs) are negative feedback control systems that synchronize the phase of a "slave" laser to that of a "master" laser, and have found applications in a variety of fields since their first demonstration in the 1960s. ${ }^{1}$ Owing to their small size, high power, large efficiency, and frequency control via injection current, the use of semiconductor lasers (SCLs) as slave current-controlled oscillators in the loop is of particular interest. A number of researchers have investigated the use of SCL OPLLs in various applications, including coherent optical communication links, ${ }^{2-4}$ clock recovery systems, ${ }^{5}$ microwave photonics, ${ }^{6}$ sensing, and coherent optical arrays. ${ }^{7,8}$ The development of SCLs with narrow linewidths in the megahertz range has led to practical systems based on SCL OPLLs.

Consider a typical heterodyne OPLL, shown in Fig. 1(a). The optical fields of the slave SCL and the master laser are mixed in a photodetector PD and the resulting error signal is amplified, downconverted using an rf offset signal, filtered, and fed back to the SCL to complete the feedback loop. When the loop is in lock, the phase of the SCL is related to the phases of the master laser and the rf offset signal by ${ }^{9}$

$\phi_{s}(t)=\phi_{m}(t)-\phi_{\mathrm{rf}}(t)$.

0091-3286/2010/\$25.00 (C) 2010 SPIE
The linearized model for the propagation of the phase in the loop, including phase noise, about the steady-state solution of Eq. (1) is shown in Fig. 1(b). The effects of the relative intensity noise (RIN) of the master and slave lasers on the phase of the locked laser are small, ${ }^{7}$ and are therefore neglected in this work. The effects of RIN on the OPLL can be minimized by using balanced detectors in the loop. $K_{S}$ is the dc open-loop gain, given by the product of the gains of the photodetector, mixer, loop amplifier, filter, and the laser response; $F_{S}(f)$ and $F_{F M}^{S C L}(f)$ are the transfer functions of the loop filter and the FM response of the SCL, respectively (normalized to have unit gain at dc); and $\tau_{S}$ is the propagation delay in the loop. $\phi_{m}(f), \phi_{r f}(f)$, and $\phi_{s}^{f r}(f)$ are (the Fourier transforms of) the phase noise of the master laser, rf offset, and the free-running slave laser respectively, and $\phi_{s}(f)$ is the phase of the locked slave laser. For simplicity of analysis, we assume that the steady-state phase error in the loop is zero; a nonzero steady-state error does not significantly change the results. The open-loop transfer function of the OPLL shown in Fig. 1, with respect to the phase of the slave SCL, is given by $^{9}$

$G(f)=\frac{K_{S} F_{\mathrm{FM}}^{\mathrm{SCL}}(f) F_{S}(f) \exp \left(-j 2 \pi f \tau_{S}\right)}{j 2 \pi f}$.

We quantify the performance of the OPLL by the variance of the residual phase noise of the OPLL, namely the phase 


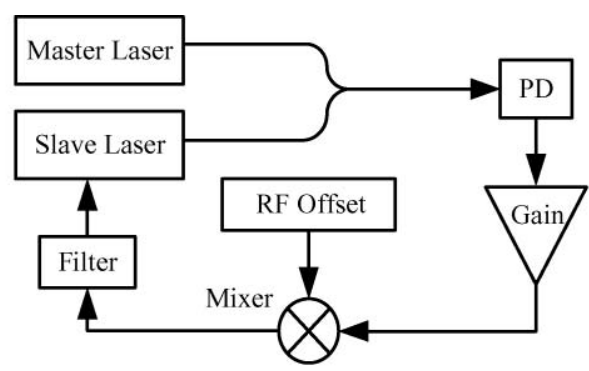

(a)

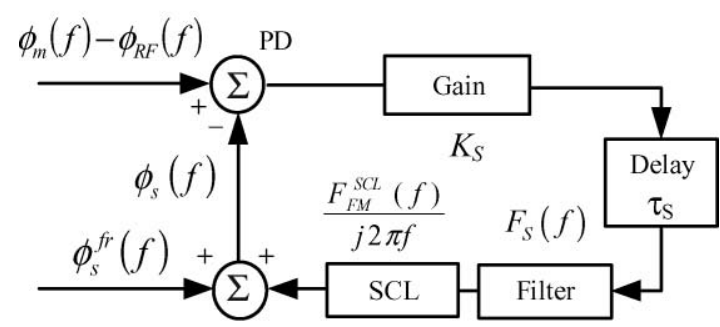

(b)

Fig. 1 (a) Schematic diagram of a hetrodyne OPLL. (b) Model for phase propagation in an OPLL, including phase noise. PD: photodetector.

difference between the locked slave and the master lasers, offset by the phase of the rf signal:

$\sigma_{e}^{2}=\left\langle\left(\phi_{m}-\phi_{\mathrm{rf}}-\phi_{s}\right)^{2}\right\rangle$.

From Fig. 1(b), the phase of the phase-locked slave laser is given by

$\phi_{s}(f)=\frac{G(f)}{1+G(f)}\left[\phi_{m}(f)-\phi_{\mathrm{rf}}(f)\right]+\frac{1}{1+G(f)} \phi_{s}^{f r}(f)$,

with $G(f)$ as defined in Eq. (2). If the master and slave lasers have Lorentzian line shapes with a summed linewidth $\Delta v$, the phase error variance can be written using the WienerKhinchin theorem as

$\sigma_{e}^{2}=\int_{-\infty}^{\infty} \frac{\Delta v}{2 \pi f^{2}}\left|\frac{1}{1+G(f)}\right|^{2} d f$,

where the integrand on the RHS represents the (two-sided) spectral density $S_{e}(f)$ of the residual phase error $\phi_{e}=\phi_{m}-$ $\phi_{\text {rf }}-\phi_{s}$ in the loop.

To achieve a low residual phase error, therefore, it is necessary to have a large loop bandwidth, defined here as the range of frequencies for which $|G(f)|>1$. From Eq. (2) and loop stability considerations, the maximum achievable bandwidth of an OPLL is ultimately limited by the loop propagation delay $\tau_{S} .{ }^{10}$ OPLLs constructed using free-space optics ${ }^{6}$ or integrated optics ${ }^{11,12}$ offer the possibility of achieving bandwidths up to a few gigahertz. However, this is based on the assumption of a well-behaved SCL FM response $F_{F M}^{S C L}(f)$. In practice, the FM response of a single section SCL exhibits a characteristic phase reversal in the frequency range of 0.5 to $5 \mathrm{MHz},{ }^{13}$ corresponding to a thermal red shift with increasing current at lower modulation frequencies and an electronic blue shift at higher frequencies. This limits the bandwidth of a negative feedback system using the SCL to only a few megahertz, which is inadequate for many OPLL applications. Several approaches to overcome this limitation

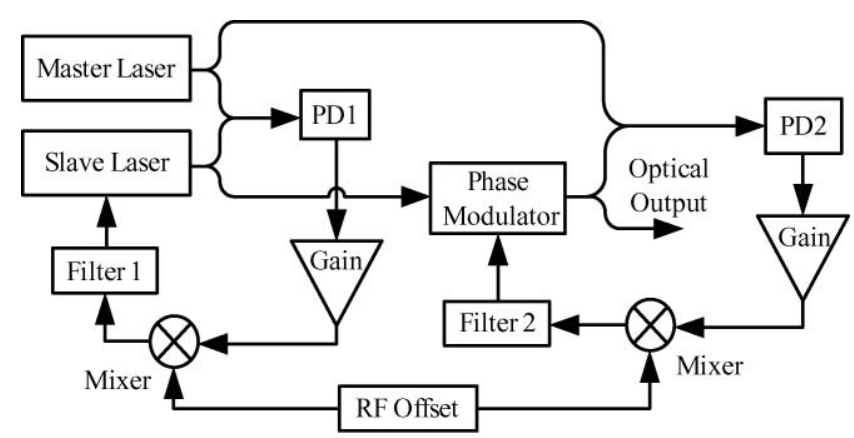

(a)

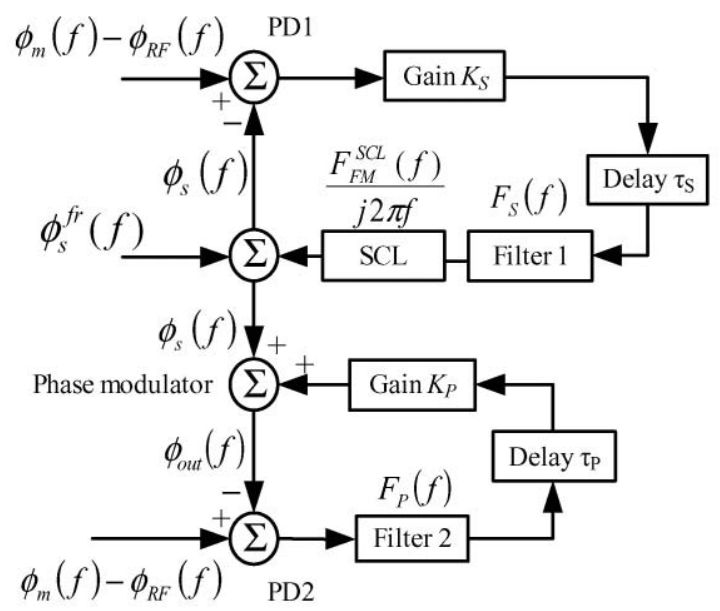

(b)

Fig. 2 (a) Schematic diagram of the double-loop configuration. (b) Linearized small-signal model for phase propagation. PD1 and PD2 are photodetectors.

have been investigated in the literature, including the use of multielectrode SCLs, ${ }^{6,11}$ the combination of phase-locking and injection-locking systems, ${ }^{14} \mathrm{FM}$ sideband locking, ${ }^{15}$ and suppressed-carrier modulation. ${ }^{16}$

In this work, we demonstrate an alternative solution that involves the use of an optical phase modulator to extend the bandwidth of the loop and reduce the residual phase error. The basic idea behind the approach is to use the phase modulator to provide correction at higher frequencies where the thermal response of the SCL is negligible. This approach eliminates the need for the design of specialized SCLs or complicated optical modulators. We demonstrate theoretically and experimentally the improvement of loop bandwidth using two different loop configurations. The use of discrete optical and electronic components in our proof-of-principle experiment results in a reduction of the residual phase noise by about a factor of two; however, the use of integrated optical phase modulators in photonic integrated circuits ${ }^{11}$ can lead to very efficient OPLL systems.

\section{System description}

\subsection{Double-Loop Configuration}

Consider the schematic diagram of the control system shown in Fig. 2(a). The SCL is first phase locked to the master laser in a heterodyne OPLL; this loop is shown with the photodetector PD1 in the figure. The output of the phase-locked SCL is phase modulated and mixed with the master laser 
in a second photodetector PD2. The resultant error signal is downconverted, filtered, and input to the phase modulator. The output of the phase modulator serves as the useful optical output. The linearized small-signal model for the propagation of the optical phase in the frequency domain is shown in Fig. 2(b). The dc gain $K_{P}$ is the product of the gains of the photodetector, mixer, loop amplifier, filter, and the phase modulator. The filter transfer function $F_{P}(f)$ is assumed to be normalized to unity.

This system can simply be analyzed as two separate feedback loops in a series. The phase $\phi_{s}(f)$ of the output of the slave laser locked to the master laser is given by Eq. (4). The open-loop transfer function of the second loop is given by

$G_{P}(f)=K_{P} F_{P}(f) \exp \left(-j 2 \pi f \tau_{P}\right)$.

The output phase $\phi_{\text {out }}(f)$ is related to $\phi_{s}(f)$ by

$$
\begin{aligned}
\phi_{\text {out }}(f)= & \frac{G_{P}(f)}{1+G_{P}(f)}\left[\phi_{m}(f)-\phi_{\mathrm{rf}}(f)\right] \\
& +\frac{1}{1+G_{P}(f)} \phi_{s}(f),
\end{aligned}
$$

which, using Eq. (4), reduces to

$$
\begin{aligned}
\phi_{\text {out }}(f)= & {\left[\frac{G_{P}}{1+G_{P}}+\frac{G}{(1+G)\left(1+G_{P}\right)}\right]\left(\phi_{m}-\phi_{\mathrm{rf}}\right) } \\
& +\frac{1}{(1+G)\left(1+G_{P}\right)} \phi_{s}^{f r},
\end{aligned}
$$

where we have omitted the argument $f$. The spectral density of the residual phase error $\phi_{e}=\phi_{m}-\phi_{\mathrm{rf}}-\phi_{\mathrm{out}}$ is therefore given by

$$
S_{e}(f)=\frac{\Delta v}{2 \pi f^{2}}\left|\frac{1}{[1+G(f)]\left[1+G_{P}(f)\right]}\right|^{2},
$$

and the variance of the phase error is

$$
\sigma_{e}^{2}=\int_{-\infty}^{\infty} \frac{\Delta v}{2 \pi f^{2}}\left|\frac{1}{[1+G(f)]\left[1+G_{P}(f)\right]}\right|^{2} d f .
$$

Comparing Eqs. (5) and (10), we see that the addition of the second feedback loop causes a reduction in the phase error at frequency $f$ by a factor $\left|1 /\left[1+G_{P}(f)\right]\right|$, and the bandwidth over which the phase noise is reduced can be extended to beyond that of the conventional OPLL, up to the propagation delay limit.

In the preceding analysis, we have made the assumption that the optical path lengths from the master laser and the phase-locked slave laser to the photodetector PD2 are equal, so that the detector is biased at quadrature (note that the OPLL forces the two optical fields at PD1 to be in quadrature). In practice, path length matching can be difficult to achieve without the use of photonic integrated circuits, and this represents a potential drawback of this approach. Further, variations in the relative optical path lengths result in changes in the gain seen by the second feedback loop, resulting in larger residual phase errors. This issue is addressed in the composite OPLL configuration discussed in the next section.

\subsection{Composite Phase-Locked Loop}

The need for precise optical path length matching is eliminated in the composite PLL architecture shown in Fig. 3(a),

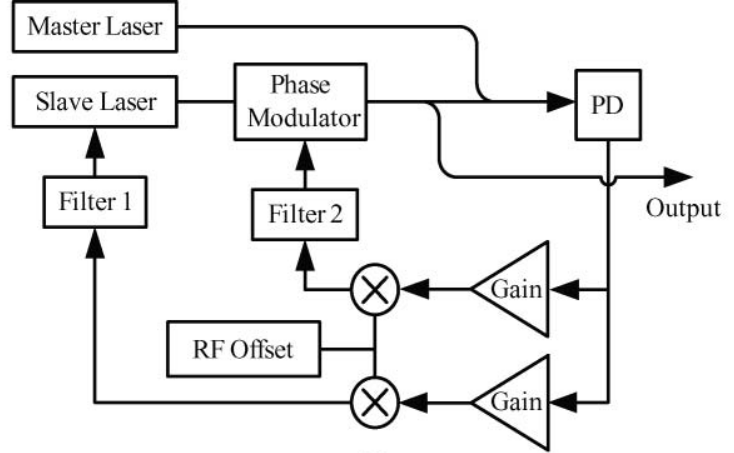

(a)

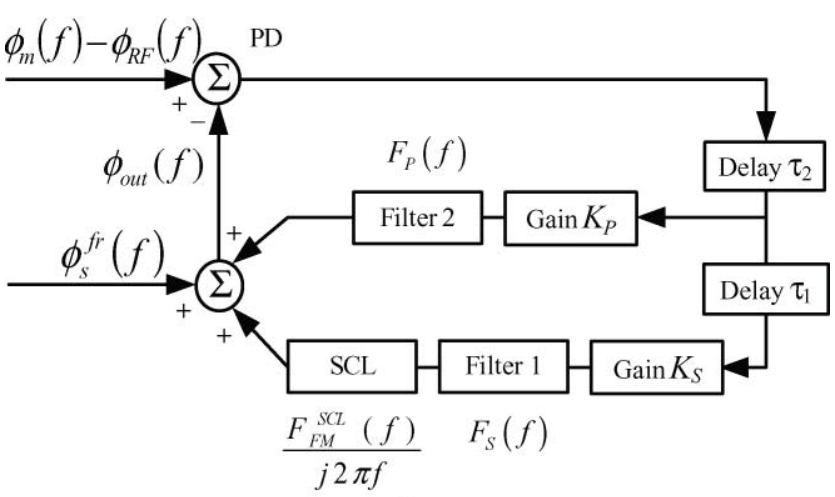

(b)

Fig. 3 (a) Schematic diagram of the composite heterodyne OPLL. (b) Linearized small-signal model for phase propagation. PD: photodetector.

where the phase error measurement is performed at a single photodetector PD. This phase error is split into two paths, one of which drives the SCL as in a conventional OPLL, whereas the second path is connected to the input of the optical phase modulator. The output of the phase modulator serves as the useful optical output. The linearized small-signal model for this composite PLL is shown in Fig. 3(b). The gain $K_{P}$ is again defined here as the product of the dc gains of the photodetector, amplifier, mixer, and filter 2. This feedback system can be regarded as comprising an integrating path (SCL) and a proportional path (phase modulator). The integral path has large gain only over a limited frequency range, but this is sufficient to track typical frequency drifts of the lasers.

Defining the open-loop transfer functions of the two feedback paths as

$$
\begin{aligned}
G(f) & =\frac{K_{S} F_{\mathrm{FM}}^{\mathrm{SCL}}(f) F_{S}(f) \exp \left[-j 2 \pi f\left(\tau_{1}+\tau_{2}\right)\right]}{j 2 \pi f}, \\
G_{P}(f) & =K_{P} F_{P}(f) \exp \left(-j 2 \pi f \tau_{2}\right),
\end{aligned}
$$

the output phase is given by

$$
\begin{aligned}
\phi_{\text {out }}(f)= & \frac{G(f)}{1+G(f)+G_{P}(f)}\left[\phi_{m}(f)-\phi_{\mathrm{rf}}(f)\right] \\
& +\frac{1}{1+G(f)} \phi_{s}^{f r}(f),
\end{aligned}
$$

and the variance of the residual phase error $\phi_{e}=\phi_{m}-\phi_{\mathrm{rf}}-$ $\phi_{\text {out }}$ is 

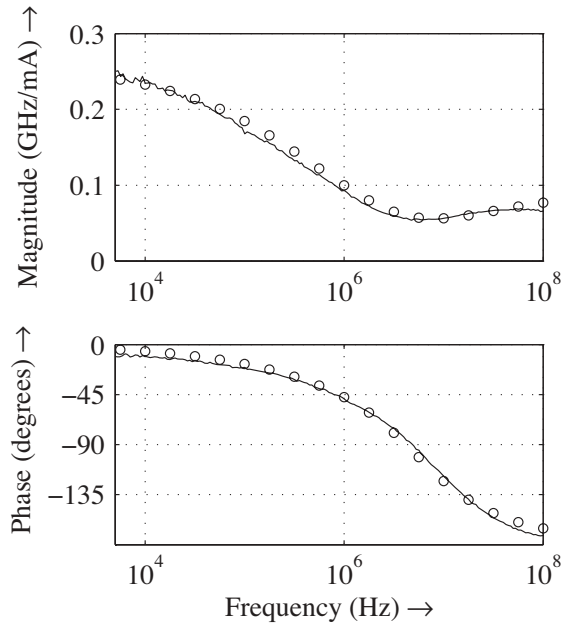

Fig. 4 Experimentally measured frequency modulation of a singlesection distributed feedback semiconductor laser (solid line) and theoretical fit using Eq. (14) (circles).

$\sigma_{e}^{2}=\int_{-\infty}^{\infty} \frac{\Delta v}{2 \pi f^{2}}\left|\frac{1}{1+G(f)+G_{P}(f)}\right|^{2} d f$

The function $G_{P}(f)$ is chosen so that at frequencies larger than the FM crossover frequency of the SCL, where the function $G(f)$ exhibits a phase reversal, the gain in the phase modulator arm $G_{P}(f)$ dominates over the gain in the SCL arm $G(f)$. This ensures phase correction over a larger frequency range, thereby leading to a reduced phase error between the output optical wave and the master laser.

\section{Results}

\subsection{Laser Frequency Modulation Response}

Two commercial single-mode distributed feedback lasers operating at a wavelength of $1539 \mathrm{~nm}$ were used in the experimental demonstration. The lasers had a 3-dB linewidth of $\sim 0.5 \mathrm{MHz}$, and their frequency modulation response exhibited the characteristic phase crossover at a frequency of $\sim 5$ $\mathrm{MHz}$, as shown in Fig. 4. The FM responses of the two lasers were very similar, and only one curve is shown for clarity.

Different models have been proposed to explain the FM response of a single-section SCL. ${ }^{13,17}$ In this work, we model the thermal contribution to the FM response of the laser using an empirical low-pass filter model,,${ }^{17}$ and the electronic response by a constant, to yield

$$
\begin{aligned}
F_{\mathrm{FM}}^{\mathrm{SCL}} & =\frac{1}{K_{t h}-K_{e l}}\left(\frac{K_{t h}}{1+\sqrt{j f / f_{c}}}-K_{e l}\right) \\
& =\frac{1}{b}\left(\frac{b-\sqrt{j f / f_{c}}}{1+\sqrt{j f / f_{c}}}\right),
\end{aligned}
$$

where $b \equiv K_{t h} / K_{e l}-1$. A good fit to experimental data is obtained with the values $b=2.7$ and $f_{c}=0.76 \mathrm{MHz}$, as seen in Fig. 4.

\subsection{Numerical Calculations}

The spectral density of the residual phase error in the loop and its variance were numerically calculated for each of the three system configurations shown in Figs. 1, 2, and 3, using Eqs. (5), (9), and (13), respectively. For the sake of simplicity, the SCL was assumed to have a Lorenzian line shape

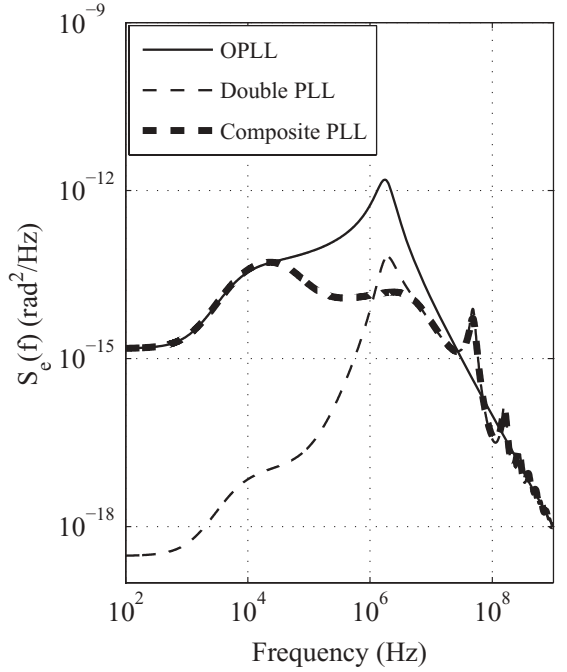

Fig. 5 Calculated two-sided spectral densities of the residual phase error in the loop, according to Eqs. (5), (9), and (13). The variance of the phase error is the area under the curves. The values of the parameters used in the calculations are listed in the text and in Table 1.

(white frequency noise spectrum) with a 3-dB linewidth of $200 \mathrm{kHz}$, and an FM response as modeled in the preceding section. The experimentally measured linewidth of the laser is larger than this value, owing to the deviation of the frequency noise spectrum from the ideal white noise assumption. ${ }^{7}$ The propagation delay in each path was assumed to be 8 ns, i.e., $\tau_{S}=\tau_{P}=\tau_{1}=\tau_{2}=8$ ns. This value was chosen to be a representative value for OPLLs constructed using fiber optics and discrete electronic components. The parameters of the loop filters were chosen to match the values of the lag filters used in the experiment. The filter transfer functions were given by

$F_{S}(f)=\frac{1+j 2 \pi f \tau_{S z}}{1+j 2 \pi f \tau_{S p}}$,

with $\tau_{S z}=24 \mu \mathrm{s}$ and $\tau_{S p}=124 \mu \mathrm{s}$; and

$F_{P}(f)=\frac{1+j 2 \pi f \tau_{P z}}{\left(1+j 2 \pi f \tau_{P p 1}\right)\left(1+j 2 \pi f \tau_{P p 2}\right)^{2}}$,

with $\tau_{P z}=15 \mathrm{~ns}, \tau_{P p 1}=1.3 \mu \mathrm{s}$, and $\tau_{P p 2}=0.8 \mathrm{~ns}$. The double pole at $1 /\left(2 \pi \tau_{P p 2}\right)=200 \mathrm{MHz}$ approximates the finite bandwidth of the op-amp used to construct the filter in the experiment.

With these parameters, the value of $K_{S}$ was optimized to result in a minimum residual phase error in the OPLL. With this optimal gain $K_{S, \text { opt }}$, the phase modulator gain $K_{P}$ was optimized to result in a minimum phase error in the double loop and composite PLL configurations. The calculated spectra of the residual phase error in the loop for the different cases are plotted in Fig. 5. The values of the optimum gain and the residual phase error calculated over an integration bandwidth of $\pm 50 \mathrm{MHz}$ are tabulated in Table 1 . It can be seen that the residual phase error is reduced by a factor of 3 to 4 due to the addition of phase modulator control.

Note that the calculated loop performance is limited by the assumed values of the propagation delay. The values used in the calculations are an order of magnitude larger than the delays that can be achieved using integrated optoelectronic 


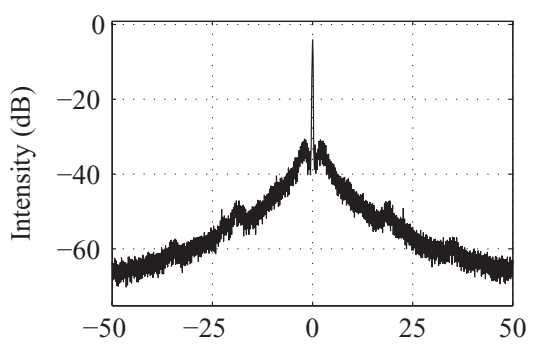

(a)

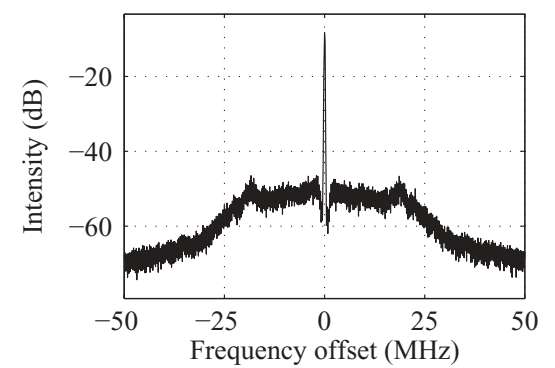

(b)

Fig. 6 Measured spectrum of the beat signal between the optical output and the master laser for an SCL in (a) a heterodyne OPLL, and (b) a double-loop feedback system shown in Fig. 2. Resolution bandwidth $=30 \mathrm{kHz}$; video bandwidth $=300 \mathrm{~Hz}$.

circuits, and therefore the residual phase error achievable in integrated OPLL circuits is expected to be much smaller. For example, in the composite PLL of Fig. 3 , if the delays $\tau_{1}$ and $\tau_{2}$ are decreased by one order of magnitude to be equal to $0.8 \mathrm{~ns}$, and if the time constants in the filter $F_{P}(f)$, namely $\tau_{P z}$ and $\tau_{P p 2}$, are correspondingly reduced by one order of magnitude, a minimum phase error of $\sigma_{e}=0.039 \mathrm{rad}$ over a bandwidth of $\pm 1 \mathrm{GHz}$ is obtained.

\subsection{Experimental Validation}

The reduction in residual phase noise was demonstrated using commercial distributed feedback lasers (JDS-Uniphase, Milpitas, California) in systems with fiber optical and discrete electronic components (Mini-Circuits, Brooklyn, New York). A fiber-coupled $\mathrm{LiNbO}_{3}$ optical phase modulator (EOSpace Incorporated, Redmond, Washington) was used in the experiments, and a narrow linewidth fiber laser (NP Photonics Incorporated, Tucson, Arizona) was used as the master laser. A rf electronic offset frequency of $1.5 \mathrm{GHz}$ was used in

Table 1 Parameters and results of the numerical calculations of OPLL performance.

\begin{tabular}{lcc}
\hline \hline & & Minimum phase error \\
System type & Optimal gain & $( \pm 50 \mathrm{MHz} \mathrm{BW})$ \\
\hline Heterodyne OPLL & $K_{S, \text { opt }}=1.4 \times 10^{8} \mathrm{~Hz}$ & $\sigma_{e}=0.43 \mathrm{rad}$ \\
Double loop & $K_{S, \text { opt }}=1.4 \times 10^{8} \mathrm{~Hz}$ & $\sigma_{e}=0.13 \mathrm{rad}$ \\
& $K_{P, \text { opt }}=71.5$ & \\
Composite PLL & $K_{S, \text { opt }}=1.4 \times 10^{8} \mathrm{~Hz}$ & $\sigma_{e}=0.12 \mathrm{rad}$ \\
& $K_{P, \text { opt }}=65.8$ & \\
\hline \hline
\end{tabular}
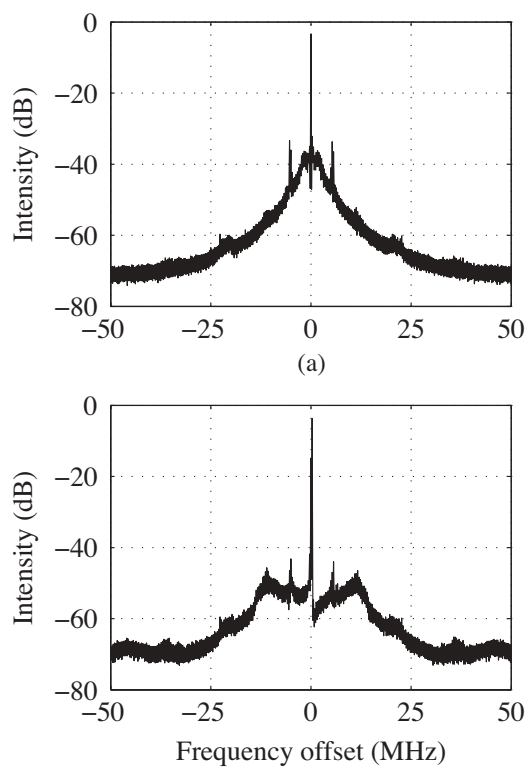

(b)

Fig. 7 Measured spectrum of the beat signal between the optical output and the master laser for an SCL in (a) a heterodyne OPLL, and (b) a composite PLL shown in Fig. 3. Resolution bandwidth $=30 \mathrm{kHz}$; video bandwidth $=300 \mathrm{~Hz}$.

the experiments. The error in the loop was calculated using the (heterodyne) beat signal between the master laser and the phase-locked optical output. When in lock, this beat signal is given by

$V_{\text {beat }} \propto \cos \left[\omega_{\mathrm{rf}} t+\phi_{e}(t)\right]$.

When the variance of the phase error $\phi_{e}$ is much smaller than $1 \mathrm{rad}^{2}$, the spectrum of the beat signal is directly proportional to the spectral density of the phase error, offset by the rf frequency. The variance of the phase error is therefore calculated by integrating the spectrum of the beat signal.

\subsubsection{Double-loop configuration}

The double-loop configuration shown in Fig. 2 was constructed with optimized loop filters $F_{S}(f)$ and $F_{P}(f)$ as given in Eqs. (15) and (16), with $\tau_{S z}=24 \mu \mathrm{s}, \tau_{S p}=124 \mu \mathrm{s}$, $\tau_{P z}=7.5 \mathrm{~ns}$, and $\tau_{P p 1}=0.66 \mu \mathrm{s}$. The measured beat signals for the OPLL and the combined double-loop system are shown in Figs. 6(a) and 6(b), respectively. A reduction in the residual phase error $( \pm 50 \mathrm{MHz}$ bandwidth $)$ from 0.31 to $0.16 \mathrm{rad}$ was measured.

\subsubsection{Composite Phase-Locked Loop}

A second, similar SCL was used in the construction of the composite PLL shown in Fig. 3. The loop filter parameters of Eqs. (15) and (16) were chosen to be $\tau_{S z}=24 \mu \mathrm{s}$, $\tau_{S p}=124 \mu \mathrm{s}, \tau_{P z}=15 \mathrm{~ns}$, and $\tau_{P p 1}=1.3 \mu \mathrm{s}$. The measured spectra of the beat signals corresponding to a conventional heterodyne OPLL using this SCL, and the composite PLL are shown in Figs. 7(a) and 7(b), respectively. The residual phase error ( $\pm 50 \mathrm{MHz}$ bandwidth) is reduced from 0.28 to $0.13 \mathrm{rad}$.

The experimentally measured reductions in the phase noise for both of the prior systems are in fair agreement with the theoretical calculations in Table 1. It must be noted that the numerical calculations are not exact and are only 
representative of the expected improvements, since nominal values for the propagation delay and the lineshape of the free-running SCL were assumed.

\section{Summary}

We propose and demonstrate experimentally that the residual phase error between the phase-locked optical output and the master laser in a semiconductor laser optical phase-locked loop can be further reduced by additional phase correction using an optical phase modulator. Feedback into the SCL is essential to compensate for frequency drifts of the SCL due to environmental fluctuations. The use of the additional phase modulator allows large loop bandwidths to be achieved, limited only by propagation delay in the system, as opposed to nonuniformities in the response of the laser. We demonstrate that the phase modulator can be used in two different configurations, both of which yield a considerable reduction in the residual phase error. The experimental demonstrations in this work use fiber optical components and discrete electronic amplifiers and mixers, which cause a large propagation delay and limit the loop bandwidths. The use of integrated photonic circuits in hybrid integrated OPLL systems using these techniques can enable bandwidths of up to a few gigahertz using standard single-section semiconductor lasers and relatively little increase in system complexity.

\section{Acknowledgments}

The authors acknowledge useful discussions with Yasha Vilenchik at Caltech.

\section{References}

1. L. H. Enloe and J. L. Rodda, "Laser phase-locked loop," Proc. IEEE 53(2), 165-166 (1965).

2. L. Kazovsky, "Performance analysis and laser linewidth requirements for optical psk heterodyne communications systems," J. Lightw. Technol. 4(4), 415-425 (1986).

3. J. M. Kahn, B. L. Kasper, and K. J. Pollock, "Optical phaselock receiver with multigigahertz signal bandwidth," Electron. Lett. 25(10), 626-628 (1989).

4. H. Philipp, A. Scholtz, E. Bonek, and W. Leeb, "Costas loop experiments for a $10.6 \mu \mathrm{m}$ communications receiver," IEEE Trans. Commun. 31(8), 1000-1002 (1983).

5. T. von Lerber, S. Honkanen, A. Tervonen, H. Ludvigsen, and F. Kppers, "Optical clock recovery methods: review (invited)," Opt. Fiber Technol. 15(4), 363-372 (2009).

6. L. N. Langley, M. D. Elkin, C. Edge, M. J. Wale, U. Gliese, X. Huang, and A. J. Seeds, "Packaged semiconductor laser optical phaselocked loop (OPLL) for photonic generation, processing and transmission of microwave signals," IEEE Trans. Microw. Theory Tech. 47(7), 1257-1264 (1999).

7. N. Satyan, W. Liang and A. Yariv, "Coherence cloning using semiconductor laser optical phase-lock loops," IEEE J. Quantum Electron. 45(7), 755-761 (2009).

8. N. Satyan, W. Liang, A. Kewitsch, G. Rakuljic, and A. Yariv, "Coherent power combination of semiconductor lasers using optical phase-lock loops," IEEE J. Sel. Top. Quantum Electron. 15(2), 240-247 (2009).

9. F. Gardner, Phaselock Techniques, 3rd ed., Wiley, New York (2005).

10. R. T. Ramos and A. J. Seeds, "Delay,linewidth and bandwidth limitations in optical phase-locked loop design," Electron. Lett. 26(6), 389-391 (1990).

11. S. Ristic, A. Bhardwaj, M. J. Rodwell, L. A. Coldren, and L. A. Johansson, "An optical phase-locked loop photonic integrated circuit," J. Lightw. Technol. 28(4), 526-538 (2010).

12. R. J. Steed, L. Ponnampalam, M. J. Fice, C. C. Renaud, D. C. Rogers, D. G. Moodie, G. D. Maxwell, I. F. Lealman, M. J. Robertson, L. Pavlovic, L. Naglic, M. Vidmar, and A. J. Seeds, "Hybrid integrated optical phase-lock loops for photonic terahertz sources," IEEE J. Sel. Top. Quantum Electron. PP(99), 1-8 (2010).
13. P Corrc, O. Girad and I. F de Faria Jr., "On the thermal contribution to the FM response of DFB lasers: theory and experiment," IEEE $J$. Quantum Electron. 30(11), 2485-2490 (1994).

14. A. C. Bordonalli, C. Walton and A. J. Seeds, "High-performance homodyne optical injection phase-lock loop using wide-linewidth semiconductor lasers," IEEE Photon Technol. Lett. 8(9), 1217-1219 (1996).

15. N. Satyan, A. Vasilyev, W. Liang, G. Rakuljic, and A. Yariv, "Sideband locking of a single-section semiconductor distributed-feedback laser in an optical phase-lock loop," Opt. Lett. 34(21), 3256-3258 (2009).

16. V. Ferrero and S. Camatel, "Optical phase locking techniques: an overview and a novel method based on single side sub-carrier modulation," Opt. Express 16(2), 818-828 (2008).

17. S. Saito, O. Nilsson and Y. Yamamoto, "Coherent FSK transmitter using a negative feedback stabilised semiconductor laser," Electron. Lett. 20(17), 703-704 (1984).

Naresh Satyan received the BTech degree in electrical engineering from the Indian Institute of Technology, Madras, Chennai, India, and the MS degree in electrical engineering from the California Institute of Technology (Caltech), Pasadena, in 2005 and 2007, respectively. $\mathrm{He}$ is currently working toward the PhD degree in electrical engineering at Caltech. His research interests include semiconductor lasers, optical phase-lock loops, optical imaging and sensing, nonlinear optics, rf photonics, and optoelectronics.

Jacob Sendowski graduated from the University of California, San Diego, with a BS degree in electrical engineering in 2007 . He received the MS degree from the California Institute of Technology in electrical engineering in 2009, where he is currently pursuing a $\mathrm{PhD}$ in the same field. His research interests include optical phaselock loops, optoelectronic swept-frequency sources, optical microresonators, photonic integrated circuits, and nonlinear optics.

Arseny Vasilyev graduated from the University of California, Davis, with a BS degree in electrical engineering and minors in physics and mathematics in 2007 . He is currently pursuing a PhD degree in applied physics at the California Institute of Technology, Pasadena. His research interests include swept-frequency semiconductor laser sources, optical imaging and sensing, optical phase-lock loops, and photonic integrated circuits.

George Rakuljic received the $\mathrm{MS}$ and $\mathrm{PhD}$ degrees in electrical engineering from the California Institute of Technology in 1983 and 1987, respectively. He is a founder of Telaris Incorporated, Santa Monica, California, and currently serves as its chief executive officer. Prior to Telaris, he founded and served as chief technology officer of Arroyo Optics, Incorporated. Previously, he was vice president of research and development at Accuwave Corporation, and a member of the research staff at the Northrop Research and Technology Center. He has numerous publications and patents in the fields of optics, materials science, and communications, and has been responsible for the development of several optoelectronic products for the telecommunications industry.

Amnon Yariv received the BS, MS, and $\mathrm{PhD}$ degrees in electrical engineering from the University of California, Berkeley, in 1954, 1956, and 1958 respectively. He joined Bell Telephone Laboratories, Murray Hill, New Jersey in, 1959 and the California Institute of Technology (Caltech) in 1964, where he is currently the Martin and Eileen Summerfield professor of applied physics and professor of electrical engineering. He took part (with various coworkers) in the first proposal and demonstration of mode-locked lasers, semiconductorbased integrated optics, phase conjugate optics, the semiconductor distributed feedback laser, GaAs optoelectronics, and in the original formulation of the theory on nonlinear quantum optics. He has published widely in the laser and optics fields, including basic texts in quantum electronics, optics, and quantum mechanics. He is a member of the American Academy of Arts and Sciences, the National Academy of Engineering, and the National Academy of Sciences. 